\title{
Uncommon sublingual ulceration in an infant
}

\section{Abdelhakim Oukerroum ${ }^{1,2}$, Fatima Zahra Elfatoiki ${ }^{2,3}$, Fouzia Hali $^{2,3}$, Faical Slimani ${ }^{1,2}$, Soumiya Chiheb ${ }^{2,3}$}

${ }^{1}$ Departement of Stomatology and Maxillofacial Surgery of UH Ibn Rochd of Casablanca, Morocco, ${ }^{2}$ Faculty of Medicine and Pharmacy, Hassan II University of Casablanca, Casablanca, Morocco, ${ }^{3}$ Department of Dermatology of UH Ibn Rochd of Casablanca, Morocco

Corresponding author: Abdelhakim Oukerroum, MD, E-mail: hakimoukerroum@gmail.com

Sir,

An eight-month-old girl was referred to our department with an extensive lingual ulceration. The parents noted that she had habitually bitten her tongue since the release of her first teeth at the age of six months. She was a poor feeder and did not sleep well because of the painful lingual ulceration. There was no family history of developmental disorders or congenital syndromes. Intraoral examination revealed a deep, circular, and extensive ulceration of the whole ventral surface of the tongue with intermittent bleeding in the tongue (Figs. la $-\mathrm{c}$ ). An examination of the rest of the intraoral mucosa revealed that the lower central incisors had recently erupted. However, there were two other ulcerations of the palmar surface of the second and third fingers caused by nocturnal finger biting. Neurological examination noted a lack of pain sensitivity related to peripheral neuropathy diagnosed as congenital insensitivity to pain.
Based on the clinical features and the particular site on the ventral surface of the tongue against the lower central incisors and ulcerative lesions of the fingers due to self-biting, the lesion was diagnosed as Riga-Fede disease. Because of the size of the ulceration, significant pain during feeding led to inadequate nutrient intake associated with permanent sleep disturbances. Radical treatment was chosen and the lower central incisors were extracted. Topical corticosteroids were prescribed to help with healing.

The term Riga-Fede disease has been used to describe a traumatic ulceration that has occurred on the ventral surface of the tongue in newborn babies and infants. It is most commonly related to neonatal or natal teeth but may also occur in infants after the eruption of the primary lower incisors [1]. This benign ulceration occurs as a result of repetitive mechanical trauma caused to the oral mucosal surfaces by the teeth and is most commonly located on the ventral surface of

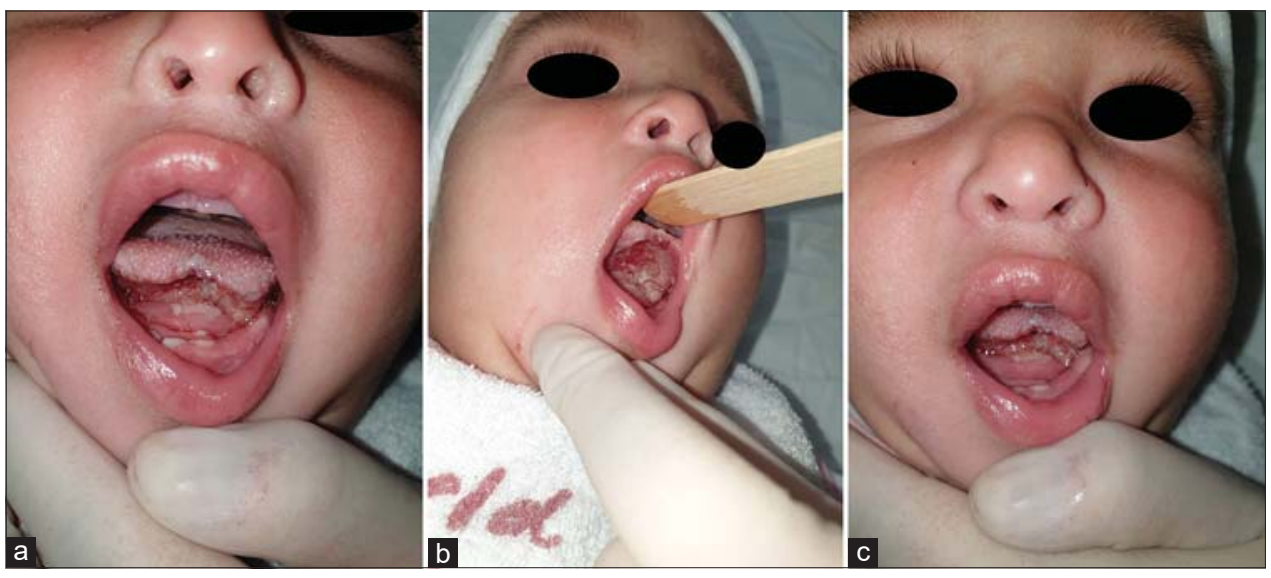

Figure 1: (a-c) The eight-month-old girl with ventral tongue ulceration caused by the primary lower anterior teeth.

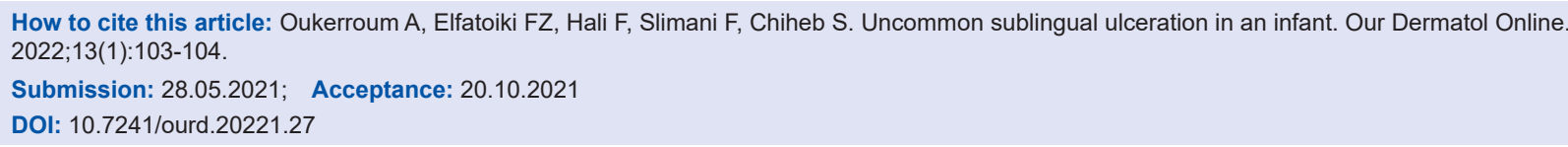


the tongue against the teeth $[1,2]$. Riga-Fede disease may reveal an underlying developmental or neurologic disorder, including congenital insensitivities to pain [3]. The case of our patient was associated with congenital insensitivity to pain.

Failure to diagnose may lead to dehydration and inadequate nutrient intake in the infant because of the significant pain during feeding. No biopsy is needed. The diagnosis of Riga-Fede disease is based on clinical characteristics $[1,2]$.

Treatment should focus on eliminating the source of trauma. Conservative treatment is attempted at first by grinding the sharp edges of the teeth and placing composite resin in a dome shape or by placing a protective ring. If conservative treatment fails to heal the wounds, radical treatment may be necessary, such as extraction of the teeth $[2,3]$.

We believe that Riga-Fede disease must be recognized by clinicians to avoid misdiagnosis and delayed treatment.

\section{Consent}

The examination of the patient was conducted according to the principles of the Declaration of Helsinki.

The authors certify that they have obtained all appropriate patient consent forms, in which the patients gave their consent for images and other clinical information to be included in the journal. The patients understand that their names and initials will not be published and due effort will be made to conceal their identity, but that anonymity cannot be guaranteed.

\section{REFERENCES}

1. Lahrichi A, El Fatoiki FZ, Hali F, Chiheb S. [Riga-Fede disease in an infant]. Ann Dermatol Venereol. 2019;146:319-320.

2. Choi SC, Park JH, Choi YC, Kim GT. Sublingual traumatic ulceration (a Riga-Fede disease): Report of two cases. Dent Traumatol. 2009;25:e48-50.

3. Yürekli A, Dinçer D. Successfully treated Riga-Fede disease. Dermatol Pract Concept. 2019;9:218-9.

Copyright by Abdelhakim Oukerroum, et al. This is an open access article distributed under the terms of the Creative Commons Attribution License, which permits unrestricted use, distribution, and reproduction in any medium, provided the original author and source are credited.

Source of Support: Nil, Conflict of Interest: None declared. 